\title{
Nonlinear Instability of an Evaporating Capillary Jet
}

\author{
Mary Saroka, ${ }^{*}$ Yi Guo, ${ }^{*}$ and Nasser Ashgriz ${ }^{\dagger}$ \\ State University of New York at Buffalo, Buffalo, New York 14260
}

\begin{abstract}
The temporal instability of an evaporating liquid jet is investigated numerically. Effects of liquid Reynolds number, disturbance wave number, and the surface evaporation rate on the growth rate of the instability are studied. The results are compared with that of the linear analytical solution. For a high evaporation rate the propagation of the disturbance is no longer linear; thus, the linear analysis cannot predict the growth accurately. A modified growth rate for the instability of an evaporating jet is defined. The breakup mechanism of viscous liquid jets and the formation of satellite drops are described. The results show that the satellite size decreases with decreasing Reynolds number and increasing wave number and evaporation rate. For large evaporation rates the satellite drops become very small, or even completely eliminated. The effect of the evaporation rate on the breakup time is also studied.
\end{abstract}

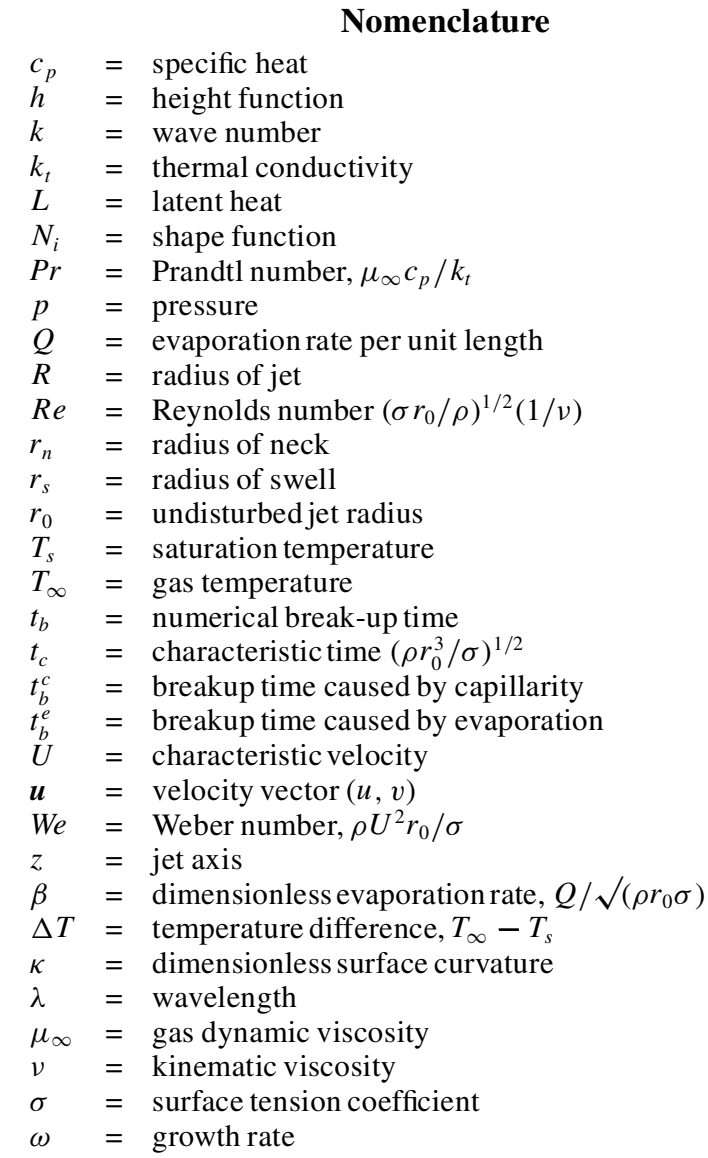

\section{Introduction}

$\mathbf{T}$ HE breakup of a liquid jet is known to be affected by a variety of disturbances such as surface displacement, pressure or velocity fluctuations, or by any variation in the temperature, viscosity or surface tension coefficient of the jet. There have been numerous studies on the instability of nonevaporating jets. ${ }^{1-19}$ However, in many instances a liquid jet is injected into a high-temperature environment, resulting in significant evaporation rates. There are very limited studies addressing the instability of an evaporating jet. Lian

Received 11 August 1999; revision received 1 March 2001; accepted for publication 30 March 2001. Copyright (C) 2001 by the American Institute of Aeronautics and Astronautics, Inc. All rights reserved.

*Graduate Student, Department of Mechanical and Aerospace Engineering.

†Professor, Department of Mechanical and Aerospace Engineering. and Reit $\mathrm{z}^{20}$ performed a linear stability analysis for an evaporating jet where the jet velocity was higher than the surface depletion rate caused by evaporation. Under these conditions the breakup of the jet was dominated by the aerodynamic interaction between the liquid and the surroundings. Their mathematical model showed that for low-speed jets in the Rayleigh regime the presence of evaporation has a destabilizing effect, whereas for high-speed jets in the atomization regime the opposite is true. Prosperetti and Plesset ${ }^{21}$ studied the stability of an evaporating horizontal planar surface of superheated liquid with infinite depth. Their analysis showed that the evaporation flux and pressure at the troughs were higher than at the crests, thus causing the liquid to squeeze into the crests and causing the crest to grow. The overall effect resulting in a thermal instability of the jet.

The purpose of this investigation is to estimate the nonlinear effects of a viscous jet with surface evaporation on 1) shape evolution, 2) breakup time, 3) growth rate of instabilities, and 4) main and satellite drop sizes after breakup. All numerical simulations were performed using the height flux method (HFM) developed by Mashayek and Ashgriz. ${ }^{22}$ This method uses the finite element method to solve the governing equations, whereas a height function method is used to represent the free surface. A brief summary of the numerical technique is provided in the next section.

\section{Mathematical and Numerical Formulation Governing Equations}

Consider a viscous liquid jet injected into an inviscid quiescent gas of temperature $T_{\infty}$. Assume the liquid jet is laminar, incompressible, and has constant properties. The gas is considered to be dynamically inert, and its temperature $T_{\infty}$ is higher than the initial temperature of the liquid. We will assume that the liquid jet has reached its saturation temperature $T_{s}$ and is evaporating at this constant temperature.

The variables are nondimensionalized by the radius of undisturbed jet $r_{0}$ and a characteristictime $\left(\rho r_{0}^{3} / \sigma\right)^{1 / 2}$. The dimensionless continuity and momentum equations are

$$
\begin{gathered}
\nabla \cdot \boldsymbol{u}=0 \\
R e \frac{\mathrm{D} \boldsymbol{u}}{\mathrm{D} t}=\nabla \cdot \boldsymbol{T}
\end{gathered}
$$

where $\boldsymbol{u}=(u, v)$ is the velocity vector and $\boldsymbol{T}=-p \boldsymbol{I}+[\nabla \boldsymbol{u}+$ $\left.(\nabla \boldsymbol{u})^{T}\right]$ is the stress tensor for a Newtonian fluid. Note that Reynolds number is defined based on the fluid properties: $R e=$ $\left(\sigma r_{0} / \rho\right)^{1 / 2}(1 / \nu)$. (In the temporal instability analysis the jet is not moving.) (D/D $t)$ is the total derivative operator and is defined as $(\mathrm{D} / \mathrm{D} t)=(\partial / \partial t)+\boldsymbol{u} \cdot \nabla$. When evaporation is taken into consideration, the stress balance on the free surface reduces to the following dimensionless boundary condition:

$$
\boldsymbol{T} \cdot \boldsymbol{n}=(\kappa / W e)\left(1+\kappa \beta^{2}\right) \boldsymbol{n} \quad \text { on the free surface }
$$




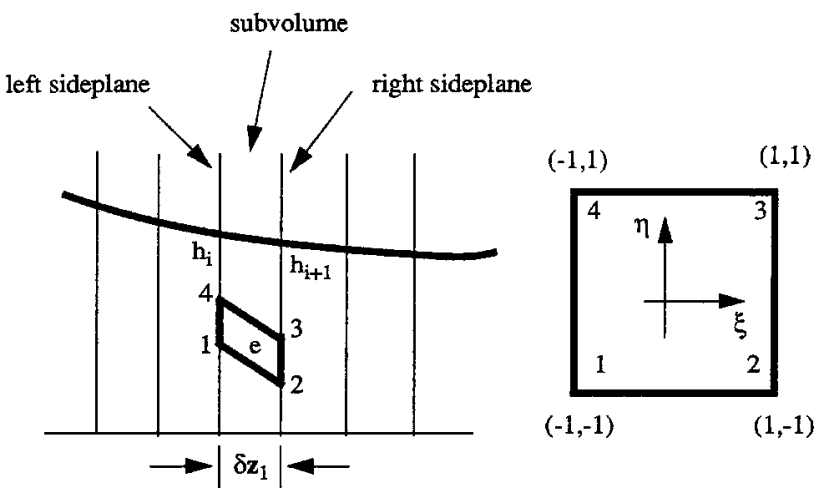

Fig. 1 Subvolume of the fluid in an axially symmetric computational domin.

As with the work of Lian and Reitz, ${ }^{20}$ the evaporation rate at the surface of the jet is modeled to be the same as for a sphere with the same curvature:

$$
Q=\left(k / C_{p}\right) \ln \left[1+\left(C_{p} / L\right) \Delta T\right]\left(1+0.276 \operatorname{Re}^{\frac{1}{2}} \operatorname{Pr}^{\frac{1}{3}}\right)
$$

To compute the surface curvature, the free surface is represented by a height function $h(z, t)$ as shown in Fig. 1. Therefore, curvature $\kappa$ is given by

$$
\kappa=h_{z z} /\left(1+h_{z}^{2}\right)^{\frac{3}{2}}-1 / h\left(1+h_{z}^{2}\right)^{\frac{1}{2}}
$$

Symmetry boundary conditions apply on the axis of symmetry and planes of $z=0$ and $\lambda / 2$ :

$$
\begin{gathered}
\frac{\partial u}{\partial r}=0, \quad v=0 \quad \text { at } \quad r=0 \\
u=0, \quad \frac{\partial v}{\partial z}=0 \quad \text { at } \quad z=0, \lambda / 2
\end{gathered}
$$

A Galerkin finite element method with penalty function formulation is used to solve Eqs. (1) and (2). Here the pressure is eliminated from the set of unknown variables by absorbing the continuity equation into the momentum equation. In this formulation the pressure is defined as

$$
p=-\Upsilon \nabla \cdot \boldsymbol{u}
$$

where $\Upsilon$ is a large number $\mathcal{O}\left(10^{9}\right)$ depending on $\mu$ and Reynolds number. ${ }^{22}$ Four-node bilinear isoparametric elements are used to approximate the velocity distribution over each element:

$$
\boldsymbol{u}(z, r, t)=\sum_{i=1}^{4} \boldsymbol{u}_{i}(t) N_{i}(z, r, t)
$$

A moving mesh is considered to discretize the computational domain. Therefore, the shape functions are time dependent. To obtain the finite element formulation, Eq. (2) is multiplied by the shape function, and integration is carried over the element volume. After the divergence theorem is invoked, the resulting equation is

$$
\begin{aligned}
\int_{\Omega(t)} & \left(N_{j} R e \frac{\mathrm{D} \boldsymbol{u}}{\mathrm{D} t}+\nabla N_{j}^{T} \cdot\left\{-p \boldsymbol{I}+\left[\nabla \boldsymbol{u}+(\nabla \boldsymbol{u})^{T}\right]\right\}\right) \mathrm{d} \Omega \\
= & \int_{\Gamma(t)} N_{j} \boldsymbol{T} \cdot \boldsymbol{n} \mathrm{d} \Gamma
\end{aligned}
$$

where $\Omega$ and $\Gamma$ are the volume and the surface area of the element, respectively. Substitution of Eqs. (3) and (7) into (9) gives the following closed-form finite element formulation:

$$
\begin{gathered}
\int_{\Omega(t)}\left(N_{j} R e \frac{\mathrm{D} \boldsymbol{u}}{\mathrm{D} t}+\nabla N_{j}^{T} \cdot\left\{\Upsilon(\nabla \cdot \boldsymbol{u}) \boldsymbol{I}+\left[\nabla \boldsymbol{u}+(\nabla \boldsymbol{u})^{T}\right]\right\}\right) \mathrm{d} \Omega \\
=\int_{\Gamma(t)} N_{j} \frac{\kappa}{W e}\left(1+\kappa \beta^{2}\right) \boldsymbol{n} \mathrm{d} \Gamma
\end{gathered}
$$

The preceding formulation is based on the Eulerian or fixed mesh where the locations of the nodes do not change with time. Special treatment of time derivatives is required when a moving grid is considered. Because the shape function is time dependent, the time derivative of velocity in discretized form becomes

$$
\left.\frac{\partial \boldsymbol{u}}{\partial t}\right|_{z, r}=\sum_{i=1}^{4} \frac{\mathrm{d} \boldsymbol{u}_{i}}{\mathrm{~d} t} N_{i}+\left.\sum_{i=1}^{4} \boldsymbol{u}_{i} \frac{\partial N_{i}}{\partial t}\right|_{z, r}
$$

The last term of Eq. (11) introduces a new convective term in Eq. (10). Here, the motion of the nodes in the $r$ direction are allowed to move according to the following simple rule:

$$
z_{i}(t+\delta t)=z_{i}(t)=\text { const., } \quad r_{i}(t+\delta t)=c r_{i}(t)
$$

where the subscript $i$ refers to the node number and $c=c(z, t)$ is a constant for each column of nodes in the radial direction defined as

$$
c=\frac{h(z, t+\delta t)}{h(z, t)}
$$

Mashayek and Ashgriz ${ }^{22}$ have shown that the total derivative of the velocity in this case becomes

$$
\frac{\mathrm{D} \boldsymbol{u}}{\mathrm{D} t}=\frac{\partial \boldsymbol{u}}{\partial t}+u \frac{\partial \boldsymbol{u}}{\partial z}+\left(v-\frac{c-1}{\delta t} r\right) \frac{\partial \boldsymbol{u}}{\partial r}
$$

Substitution of $\mathrm{D} \boldsymbol{u} / \mathrm{D} t$ from Eq. (14) into Eq. (10) concludes the finite element formulation of the problem.

\section{Free-Surface Determination}

The free surface of the jet is not known a priori and is determined using the HFM developed by Mashayek and Ashgriz. ${ }^{22}$ Consider the liquid domain shown in Fig. 1 with a free surface at the top. The domain is divided into several vertical subvolumes of width $\delta z_{I}$ and volume $V_{I}$. The location of the free surface on the left and right sides of this subvolume is given by $h_{i}$ and $h_{i+1}$ at time $t$, respectively. Knowing the velocity distributions over the sideplanes, the location of the free surface is found, i.e., $h_{i}$ and $h_{i+1}$ at time $t+\delta t$. This is achieved by first calculating the net flux of the fluid passing through the sideplanes during the time interval $\delta t$ and by determining the new volume of fluid at $t+\delta t$ in the subvolume by adding the net flux to the previous volume at time $t$. Then the interface, which is located in any two neighboring subvolumes, is approximated by a line segment defined by $h=a z+b$, where $a$ and $b$ are two constants to be determined from the known volumes.

Briefly, the technique is implemented as follows. The initial fluid domain is discretized into subvolumes, and the volume of fluid in each subvolume is calculated. Then, the free surface is recovered from this set of numbers (subvolumes) using the line segment approximation. A finite element mesh is then generated based on the free surface. The flow equations are solved, and the velocity field is calculated. The new subvolumes are then found by calculating the flux of the fluid passing through the sideplanes. In the final step the new free surface is reconstructed based on these numbers, and a new finite element mesh is generated. This completes the sequence of the operations needed to advect and reconstruct the fluid surface. For the evaporating jets the volume of the fluid in each surface cell is modified using the evaporation rate. The evaporation rate is multiplied by the time interval in each time step, and the resulting volume is subtracted from the volume of fluid in each surface cell. The new surface is reconstructed based on the new volume fractions after the evaporation.

All numerical simulations were performed using four elements in the radial direction, whereas the number of elements used in the axial direction were varied depending on the wave number. (The effect of the grid resolution on the accuracy of these simulations is discussed in Ref. 19.) Specifically, 40 elements were used for a wave number of $k=0.7$, and more were used as the wave number decreased until 100 elements were used for the smallest wave number of $k=0.2$. In accordance with the numerical method, the nodes were equally spaced in the axial direction, whereas the node spacing was allowed to change as a result of surface deformation. 
Temporal Instability of an Evaporating Jet

In this study the cylindrical liquid jet is initially at rest with a cosine-shaped spatially harmonic surface displacement:

$$
r=R-\epsilon_{0} \cos (k z)
$$

The wave number $k=2 \pi R / \lambda$ and $R$ are determined such that the volume of the jet is kept constant when the initial amplitude $\epsilon_{0}$ is changed. Therefore,

$$
R=\left(1-\epsilon_{0}^{2} / 2\right)^{\frac{1}{2}}
$$

Use of symmetry allows the jet to be modeled using only half a wavelength of a cosine function. The trough of the initial surface is set at $z=0$ and the crest at $z=\lambda / 2$. Hereinafter, the "initial" crest of the sinusoidal surface is referred to as the swell point, and its trough is referred to as the neck point. The dynamics of this jet caused by the combined capillary and evaporation effects is investigated for various values of initial disturbance wave number $k$, the jet Reynolds number $R e$, and the evaporation rate $\beta$ defined as $Q / \sqrt{ }\left(\rho r_{0} \sigma\right)$, where $Q$ is the evaporation rate per unit lenght of the jet as defined in Eq. (4).

In this investigation a jet with a zero evaporation rate is governed solely by capillary forces and is referred to as a nonevaporating jet. Evaporating jets with small rates (i.e., $\beta=10^{-5}$ ) to high rates (i.e., $\beta=10^{-1}$ ) are considered for three Reynolds numbers of 1,10 , and 100 and for various wave numbers. To put the evaporation rates into perspective, a moderate rate of $\beta=0.025$ represents water in an environment of $T_{\infty}=470 \mathrm{~K}$, whereas a rate of $\beta=0.05$ represents water in an environment of $T_{\infty}=750 \mathrm{~K}$.

\section{Surface Evolution}

Details of the shape evolution of a liquid jet with $R e=1,10$, and 100 and $k=0.2$ and 0.7 for both a nonevaporating $(\beta=0.0)$ and evaporating $(\beta=0.05)$ surfaces are presented in Figs. 2 and 3 , respectively. The calculations were stopped when the radius of the jet reached its minimum point of $1 \%$ of the undisturbed jet radius, i.e., $0.01 r_{0}$. This point is defined as the breakup point. Comparison of the surface evolutions for the nonevaporating vs the evaporating jet reveals the effect the mass depletion has on the characteristics of the breakup. For liquid jets with no evaporation (Figs. 2a-2c and $3 a-3 c$ ), the breakup point migrates toward the swell region as the Reynolds number increases. With increased Reynolds number the rapid fluid convection forces the fluid away from the neck. Because the mass of the liquid must be conserved, the displacement of this fluid causes a thin ligament to form while simultaneously increasing the swell region. This minimum point eventually breaks, resulting in the formation of a satellite drop. Conversely, for the evaporating jet (Figs. $2 \mathrm{~d}-2 \mathrm{f}$ and $3 \mathrm{~d}-3 \mathrm{f}$ ), the breakup point is at the neck point of the initial disturbance, retaining approximately a sinusoidal profile for the lifetime of the jet. Regardless of the Reynolds number, the displacement of the liquid becomes much more difficult because of the presence of the mass depletion at the surface. Therefore, the capillary disturbances grow locally. Consequently, the presence of evaporation reduces the size of the satellite drop until at some critical evaporation rate no satellite drop is formed. Additionally, the amount of time for the breakup to occur is affected by the evaporation rate. For nonevaporating jets the breakup time decreases as the Reynolds number is increased. For an evaporating jet the breakup time 1) decreases for all cases as the evaporationrate is increased and 2) is essentially independent of the Reynolds and wave number for higher evaporations rates. Breakup times will be discussed further in a later section.

\section{Time Evolution of the Amplitudes}

For a nonevaporatingliquid jet Ashgriz and Mashayek ${ }^{19}$ showed that the growth rate could be found by fitting a straight line to the logarithmic difference between the swell and the neck radii. Their numerical solution of the nonlinear governing equations yielded results that are very close to those of the analyticalresults for small amplitude disturbancesand low Reynolds numbers. In an effort to quantify the growth rates for evaporating jets, similar logarithmic curves were plotted for various evaporation rates for the range of Reynolds numbers and wave numbers considered in this investigation.

Figure 4 shows the logarithmic variations of the amplitude of the neck $\left|r_{n}-R\right|$, the swell $\left|r_{s}-R\right|$, and their difference $\left|r_{s}-r_{n}\right|$ for a jet with $R e=10, W e=1, k=0.7$, and $\epsilon_{0}=0.05$ as a function of four different evaporation rates. For a low evaporation rate of $\beta=10^{-5}$ (Fig. 4a), the logarithmic difference between the swell and neck can be approximated by a straight line if the initial and final periods are neglected. Therefore, for the majority of the jet lifetime a growth rate (i.e., the slope of the line) can be specified. Figure $4 b$

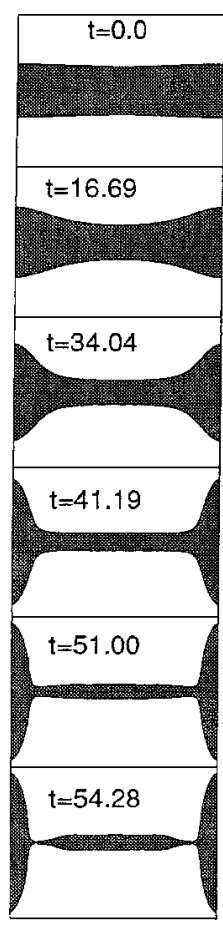

a)

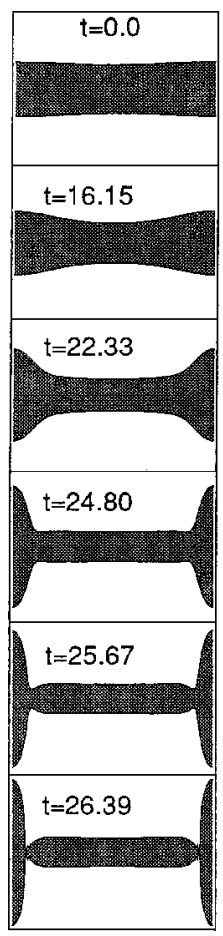

b)

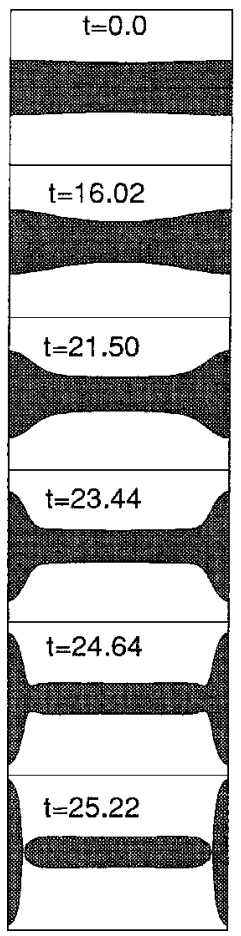

c)

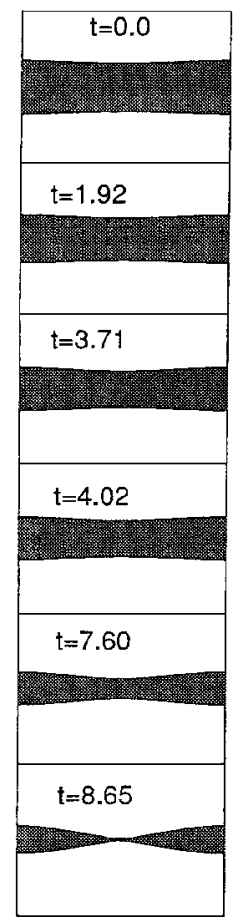

d)

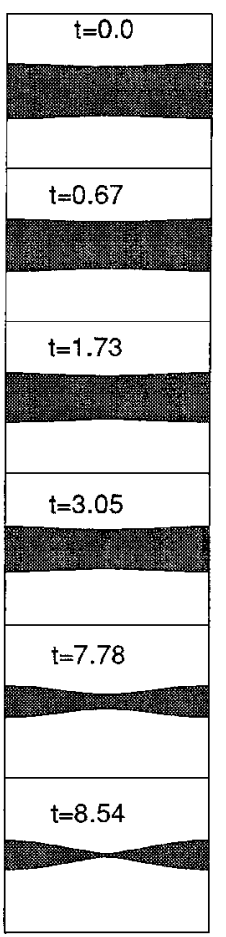

e)

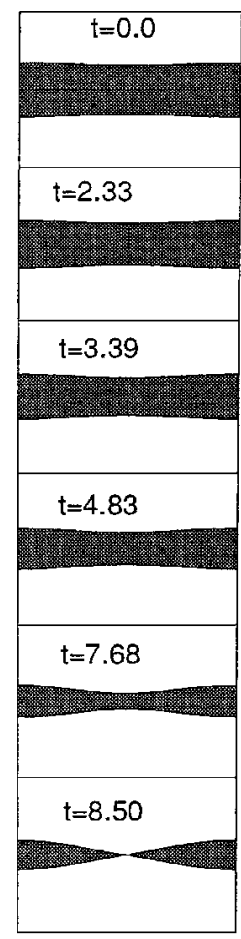

f)

Fig. 2 Surface evolutions for $k=0.2$. $\beta=0$ : a) $R e=1$, b) $R e=10$, and c) $R e=100$. $\beta=0.05$ : d) $R e=1$, e) $R e=10$, and f) $R e=100$. 


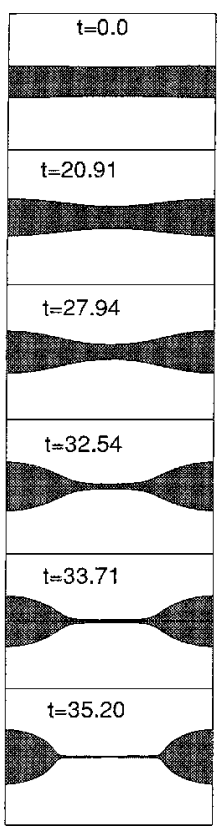

a)

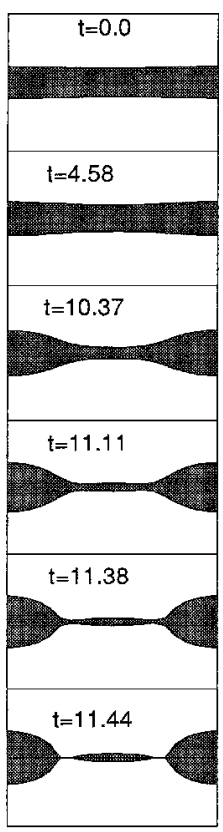

b)

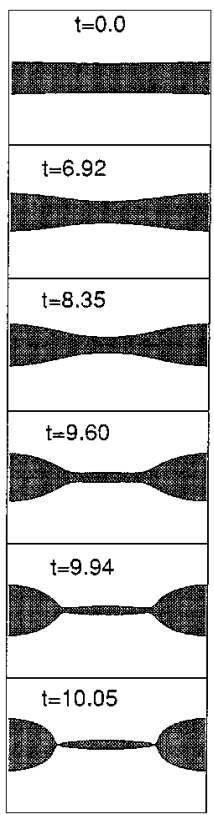

c)

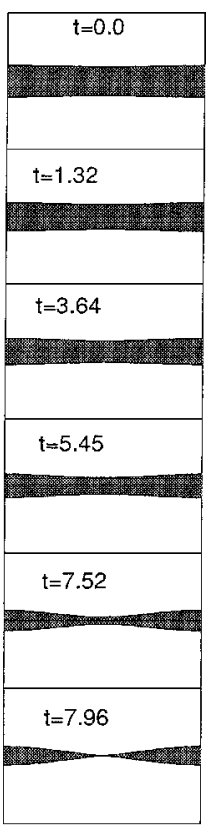

d)

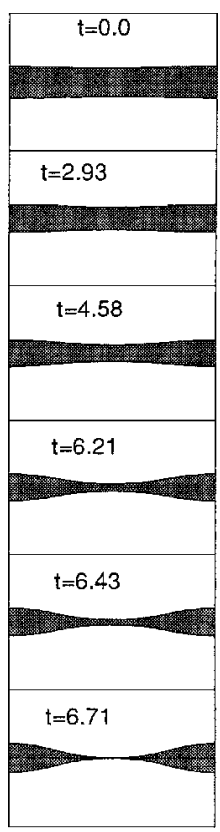

e)

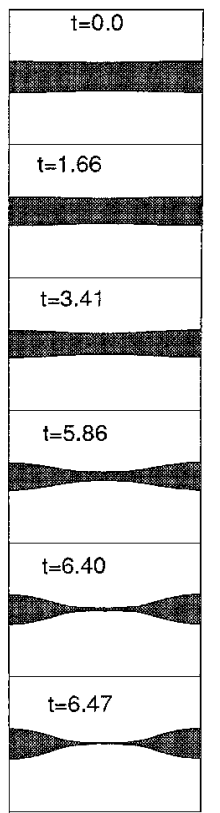

f)

Fig. 3 Surface evolutions for $k=0.7$. $\beta=0$ : a) $R e=1$, b) $R e=10$, and c) $R e=100$. $\beta=0.05$ : d) $R e=1$, e) $R e=10$, and f) $R e=100$.

a)
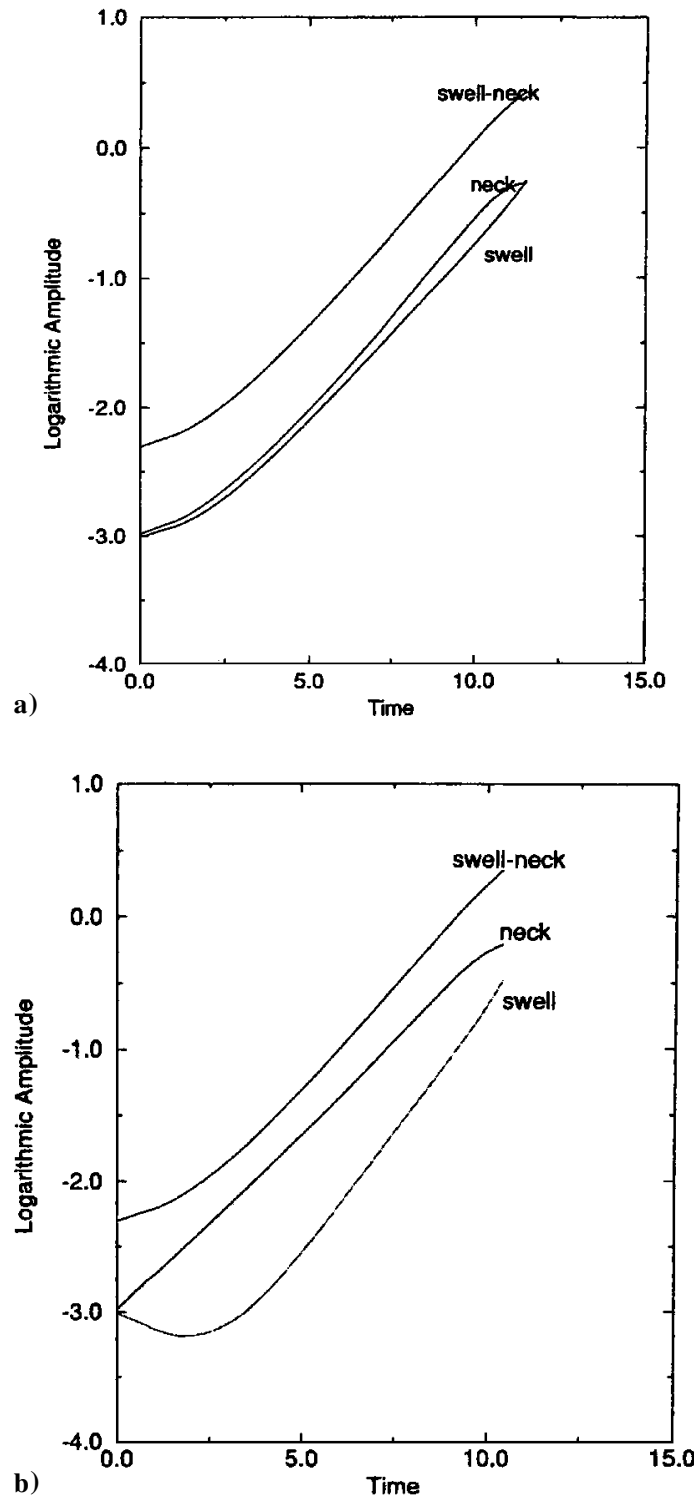
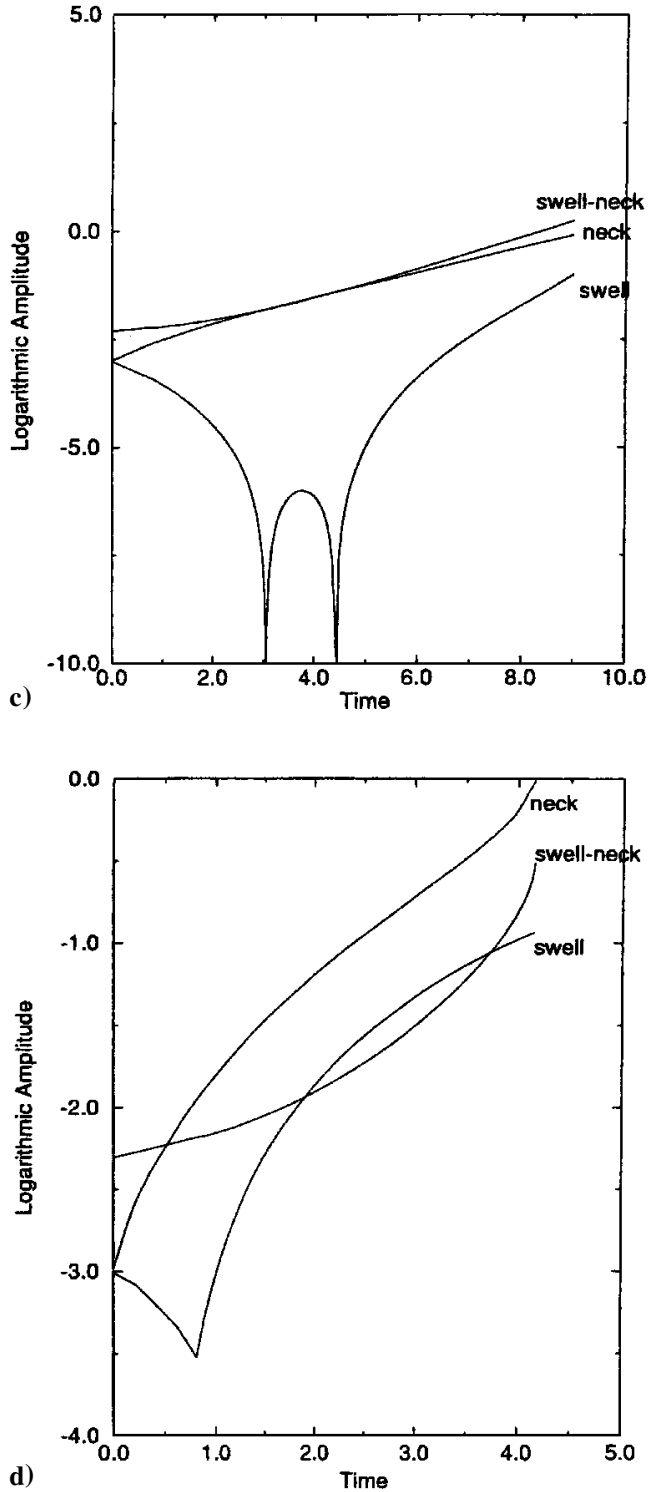

Fig. 4 Logarithmic variation of the amplitudes of the swell, neck, and their difference for a) $\beta=0, b) \beta=0.01, c) \beta=0.025$, and d) $\beta=0.1$. 
depicts the logarithmic amplitude variations for the same jet, this time with an evaporation rate of $\beta=0.01$. Unlike the preceding case, the logarithmic amplitude at the swell point first decreases slightly before it begins to increase. This behavior is attributed to the fact that prior to the velocity field becoming fully developed the evaporation at the surface dominates the surface evolution of the jet. Once the velocity field has developed, the surface tension effect quickly compensates, and at that point the curve begins to increase. The neck radius, on the other hand, always decreases. Even with the slight swell reduction increase, the logarithmic difference is still essentially linear for the majority of lifetime of the jet. As the evaporationrate is further increased to $\beta=0.025$ (Fig. 4c), the swell exhibits fluctuations in its amplitude. First, the evaporation at the surface dominates, causing a reduction until the radius balances with $R$, where $\left|r_{s}-R\right|$ approaches $-\infty$. After this point the surface tension dominates, causing the swell to grow until the evaporation once again dominates causing another reduction in swell radius. The swell radius again balances with $R$, causing a second $-\infty$ spike, at which point the surface tension completely dominates for the remainder of the jet's lifetime. By this time the velocity field is fully developed, thus allowing surface tension to dominate over the evaporation causing the swell region to continuously increase until the jet breaks. Figure $4 d$ represents the logarithmic variations when the evaporation rate is $\beta=0.1$. As with the preceding two cases, the swell radius begins to decrease because of the evaporation effect. In this case, however, the radius experiences a dramatic shift in direction earlier in the jet's lifetime. At this high evaporation rate the phenomenon is highly nonlinear (none of the curves can be fitted with a straight line), and the effect of both the capillary and evaporation mechanisms is strongly coupled.

To gain a better understanding of the effect of the nonlinearitieson the jet breakup and the mode coupling, the jet surface is decomposed into its linear mode by implementing the Fourier expansion:

$$
r(z, t)=R+\sum_{n=0}^{\infty} c_{n} \cos (n k z)
$$

The orthogonality of the cosine functions and numerical integration is used to determine the coefficients $c_{n}$. Figure 5 shows the amplitude of the fundamental, zeroth, second, third, and fourth harmonics of the initial disturbancewith time for the case given in Fig. 4. Figure 5a represents the harmonics for the jet with virtually no evaporation, $\beta=10^{-5}$. Here the jet becomes unstable because of the growth of the fundamental, $n=1$, disturbance, $k=0.7$. For this wave number the higherharmonics are stable, e.g., $2 k=1.4$, and thus do not grow until close to the breakup time. Even as the evaporation rate is increased, similar behavior for both the fundamental and higher harmonics is observed. For $\beta=0.1$ (Fig. $5 \mathrm{~d}$ ) the higher harmonics have very small growth. The most effected harmonic is the zeroth. This means that as the evaporation rate is increased the location of the axis of the surface disturbance has shifted and thus explains why the jet shape becomes more and more sinusoidal.

\section{Dispersion Curves}

Plots of the growth rates for various wave numbers are given in Fig. 6. The actual calculated points are marked. The curves are only fits to the calculated points. Growth rates were determined only for the cases where a linear fit to the logarithmic value of the difference between the swell and neck was possible. Consistent with linear theory, the presence of viscosity reduces the magnitude of the growth rates for all wave numbers when the evaporationrate was zero. However, as the evaporation rate is increased, the growth rates increase for similar Reynolds numbers. As a result, the presence of evaporation actually hinders the effect of viscosity, allowing the instabilities to grow at a faster rate. The overall amount of viscous damping is still most effective at the larger wave numbers resulting in maximum growth rates at the same wave numbers as the nonevaporating cases.

For the range of Reynold numbers considered in this investigation, it is seen that as the evaporation rates increase the instabilities become increasingly nonlinear. Lian and Reitz ${ }^{20}$ used their linear model to calculate the growth rates for a high-Reynolds $(R e=$
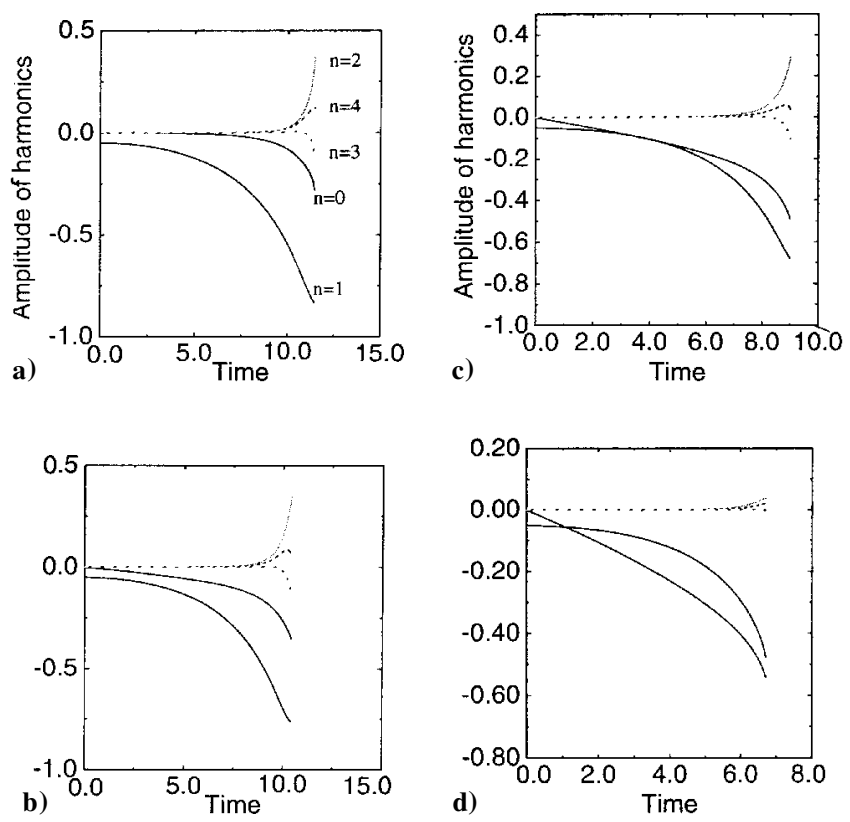

Fig. 5 Amplitude of the fundamental (—), zeroth (-), second $(\cdot \cdots)$, third $(\cdots)$, and fourth $(---)$ harmonics as a function of time; $R e=10, k=0.7, \varepsilon=0.05$; a) $\beta=0$, b) $\beta=0.01$, c) $\beta=0.025$, and d) $\beta=0.05$.
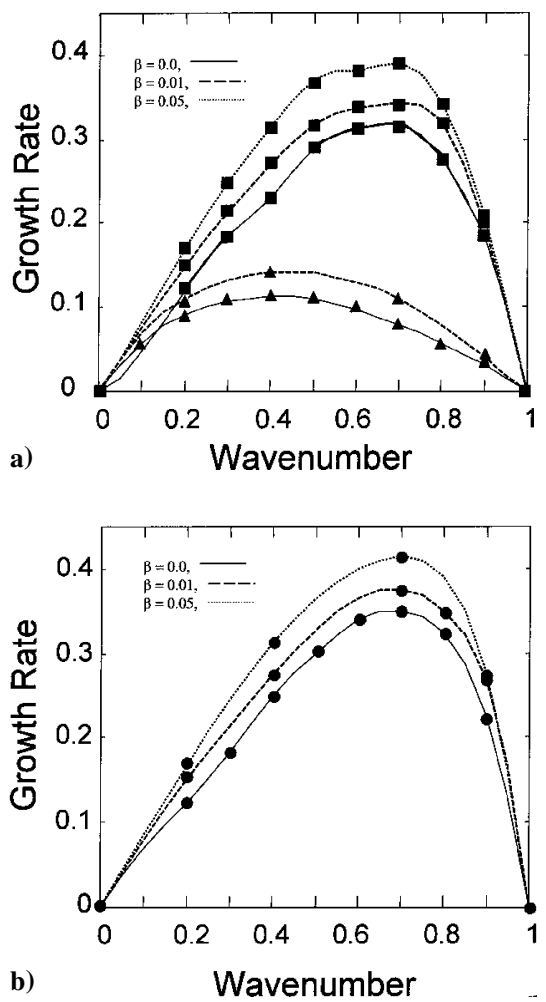

Fig. 6 Numerically calculated growth rates (symbols) for defferent evaporation rates: a) $\triangle, R e=1$ and $\square, R e=10$; and b) $\bullet, R e=100$.

$\left.2 \times 10^{6}\right)$ - and Weber-number $\left(W e=4 \times 10^{4}\right)$ jet for various evaporation rates and wave number. Using their initial conditions, three simulations for the same Reynolds and Weber number jet, along with $k=0.7$, were simulated numerically. The first case was for a nonevaporating jet, whereas the other two were for an evaporating jet with $\beta=3 \times 10^{-6}$ and $5 \times 10^{-6}$, respectively. Their linear model yielded growth rate values of $0.36,0.42$, and 0.59 for each case. When their results were compared to the results obtained numerically, only the nonevaporatingcase provided a good match. In fact, to compute the growth rates close to their values the evaporation rates were found to be $3.0 \times 10^{-3}(\omega=0.41)$ and $3.0 \times 10^{-2}(\omega=0.56)$, 
respectively. Therefore, the effect of evaporation introduces nonlinearities in which linear theory is invalid for high-Reynolds- and Weber-number jets.

\section{Breakup Time}

Two different mechanisms can affect the stability of an evaporating liquid jet. The first is caused by capillarity, and the second results from mass depletion by evaporation. Individually, each effect can be characterized by considering the amount of time it takes for the jet to break up. Therefore, the time it takes a jet to break up because of only capillary forces is defined to be $t_{b}^{c}$, whereas the breakup time for a jet with only evaporation is defined to be $t_{b}^{e}$. The latter time representing the time it takes to evaporate a cylindrical jet of radius $r_{0}$ without any surface deformation. When both mechanisms are acting on the jet as is the case for our numerical simulations, the breakup time is defined to be $t_{b}$.

The capillary breakup time $t_{b}^{c}$ can either be directly calculated by a nonlinear simulation, such as that of Ashgriz and Mashayek, ${ }^{19}$ or approximated using the linear theory of Rayleigh ${ }^{3}$ for inviscid jets or of Chandrasekhar ${ }^{5}$ for viscous jets. Based on linear viscous theory, the breakup time can be computed as follows:

$$
t_{b}^{c}=(1 / \omega) \ln \left(R / \epsilon_{0}\right)
$$

The actual breakup time $t_{b}^{c}$ is a strong function of the Reynolds number, and for a low Reynolds number the inviscid solution does not provide a good approximation of the actual breakup time.

If only evaporation is present, an estimate for the breakup time $t_{b}^{e}$ can be computed by dividing the product of an undisturbed jet's surface area and density by the evaporation rate per length:

$$
t_{b}^{e}=\rho \pi r_{0}^{2} / Q
$$

where $Q=\beta \sqrt{ }\left(\rho r_{0} \sigma\right)$. Solving in terms of the dimensionless evaporation rate yields

$$
t_{b}^{e}=(\pi / \beta)\left(\sqrt{\rho r_{0}^{3} / \sigma}\right)
$$

If the evaporation time is much smaller than the capillary time, i.e., $t_{b}^{e} \ll t_{b}^{c}$, there is no time for the capillary effects to develop, and the jet dynamics are governed by pure evaporation. Therefore, if the jet has a sinusoidal disturbance the shape of that disturbance remains the same throughout the jet lifetime. On the other hand, if $t_{b}^{e} \gg t_{b}^{c}$ the jet instability is governed by capillary effects, and the mass loss is not significant enough to change this behavior. If $t_{b}^{e} \sim t_{b}^{c}$, both mechanisms are comparable.

The nondimensional breakup times for various evaporation rates for a jet with $R e=10$ and for $k=0.2,0.4,0.5,0.6$, and 0.7 are shown in Fig. 7. In all cases an increase in evaporation rate substan-

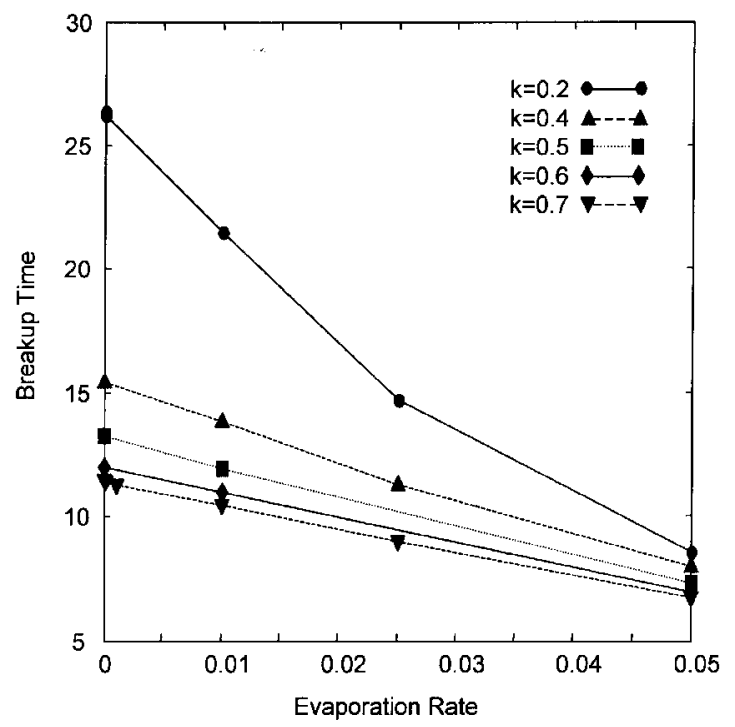

Fig. 7 Variation of breakup times with evaporation rate for $R e=10$ and various wave numbers. tially reduces the breakup time. For lower wave numbers the change in breakup time is much faster than for the higher wave numbers. This can be explained by considering the growth rates and the effect of evaporation simultaneously. At low wave numbers, although the jet is unstable, the growth rate is very slow, and as a result the mass depletion has a greater effect. The overall effect results in a faster reduction of the jet's radius and thus faster breakup times. As the growth rates increase, the effect on evaporation is partially neutralized, causing the change of breakup times to be reduced. This is clearly seen as the wave numbers are increased in Fig. 7. The effect of Reynolds number with increasing evaporation rate is shown in Fig. 8. Here, the logarithmic evaporation rates are plotted for the three Reynolds numbers with a low wave number of 0.2 and a high wave number of 0.7 . Generally, as the Reynolds number is increased, the breakup time decreases, regardless of the wave number or evaporation rates. However, when the effect of evaporation is taken into consideration the most dramatic changes are seen for small Reynolds numbers. Again, this can be attributed to the small growth rates. Substantial changes in breakup time are observed once the evaporation rate becomes $\beta \geq 0.01$. Therefore, once this rate is obtained the evaporation is completely dominant, regardless of the growth rates, resulting in similar breakup times of $<10$ for all Reynolds and wave numbers.

Using Eqs. (18) and (19), a comparison between the governing mechanism in relation to the numerical results can be made. When the evaporation rate is zero, the jet is governed solely by capillary effects. The breakup times for a nonevaporating jet match those computed by Ashgriz and Mashayek ${ }^{19}$ for a nonlinear capillary jet. For nonzero evaporation rates Table 1 provides a comparison of the numerically computed breakup times vs those for a jet governed solely by evaporation [Eq. (19)].

Only for a very small evaporation rate, i.e., $\beta=10^{-4}$, does the capillary force have time to dominate before the evaporation rate has any effect. This seems consistent with the numerical breakup times computed. As the evaporation rate increases, i.e., $\beta \geq 0.01$,

Table 1 Comparison of evaporation breakup times with the numerical results for a 1-mm water jet at the wave number $k=0.7$

\begin{tabular}{lcccc}
\hline \hline & $\begin{array}{c}\text { Eq. (19) } \\
t_{b}^{e}\end{array}$ & $\begin{array}{c}\text { Numerical } \\
\beta e=1\end{array}$ & $R e=10$ & $R e=100$ \\
\hline 0 & $\infty$ & 35.2 & 11.44 & 10.1 \\
$10^{-4}$ & 116.3 & 33.5 & 11.38 & $-\overline{8.25}$ \\
0.01 & 1.163 & 18.7 & 10.4 & - \\
0.025 & 0.465 & 13.2 & 8.99 & $\overline{6.47}$ \\
0.05 & 0.233 & 7.96 & 6.71 & 6.45 \\
\hline \hline
\end{tabular}

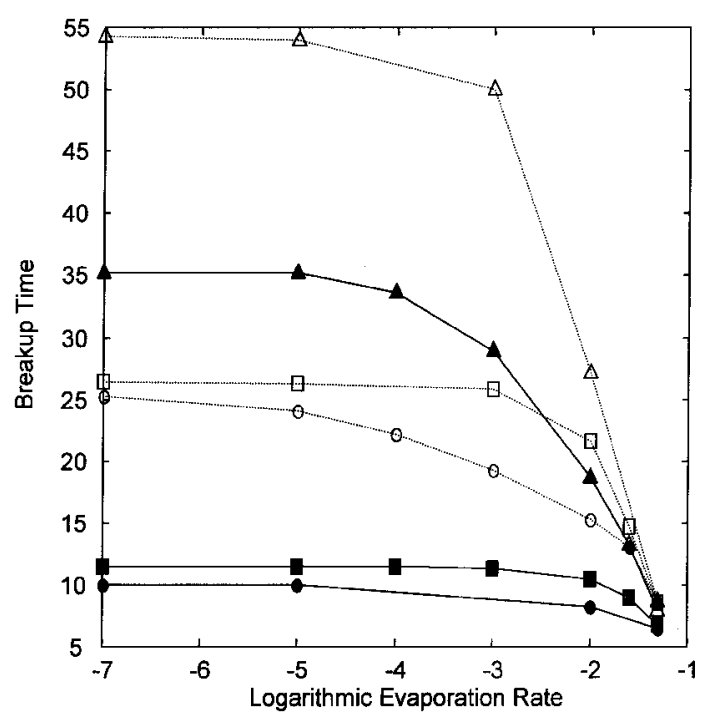

Fig. 8 Variation of breakup times with logarithmic evaporation rate for $\operatorname{Re}=1(\triangle, \triangle)$; $R e=10(\square, \square)$; and $R e=100(\bullet$, O). Open Symbols are for $\boldsymbol{k}=\mathbf{0 . 2}$, and closed symbols are for $\boldsymbol{k}=\mathbf{0 . 7}$. 


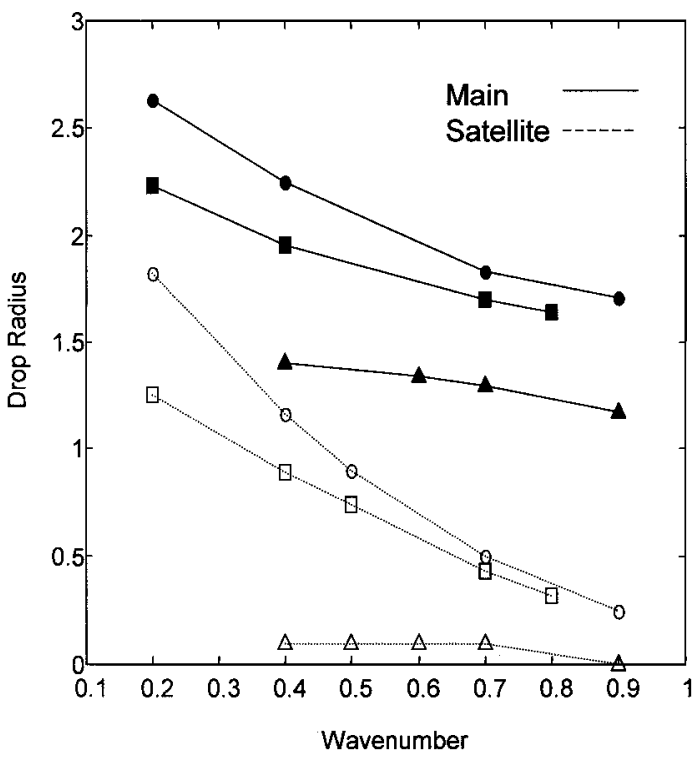

Fig. 9 Main $(\bullet, \square, \Delta)$ and satellite $(0, \square, \triangle)$ drop radii for $\boldsymbol{R e}=\mathbf{1 0}$ and $k=0.7$ for three evaporation rates: $\bullet, \beta=0 ; \square, \beta=0.01$; and $\Delta$, $\beta=0.05$.

the time it would take a jet to completely evaporate is much smaller than it would take for breakup to occur because of capillarity. This is also confirmed numerically as evidence by the faster breakup times. To compute a critical evaporation rate, which is defined to be when both mechanisms are comparable, Eq. (19) and the capillary time are used. In doing so, for a jet with $R e=1,10$, and 100, this evaporation rate would be $0.00033,0.001$, and 0.0012 , respectively. Therefore, even moderate evaporation rates will begin to have an effect on the jet's final breakup time.

\section{Main and Satellite Drop Size}

A deviation from linear theory results in the formation of a ligament between the two swell regions. As the breakup point migrates toward the swell region, it eventually breaks allowing the ligament to break free. Surface tension will then dominate, thus pulling the ligament into a sphericaldrop. In this investigation the volume of the ligament was used to compute the radius of an equivalent spherical satellite drop, while the volume of the swell was used to compute the radius of the main drop. Note, however, because the calculations were stopped once the jet reached its breaking point, any effect the evaporation would have on the ligament/swell as it contracted was not taken into consideration. Therefore, the actual sizes of the satellite and main drops would be slightly smaller than the results presented here. Figure 9 shows the computed main and satellite drop radii for $R e=10$ and various wave numbers. As expected, the overall effect of evaporation reduces the drop sizes as the effect is increased. In fact, once the evaporation rate became greater than 0.05 no satellites were observed for the range of Reynolds and wave numbers considered. For evaporating jet with $R e=1$ the satellite drop sizes were almost zero, and for $R e=100$ they were very close to that of the $R e=10$.

\section{Conclusions}

We have used a Galerkin finite element method in conjunction with the height-flux method to investigate numerically the effect evaporation has on the instabilities of a capillary liquid jet. The finite element method was used to solve the governing equations, whereas the height-flux method was used for the advection and reconstruction of the free surface.

It is observed that the mass depletion at the jet's surface as a result of the evaporation results in a remarkable change in breakup con- figurations and breakup time. The surface evaporation has a large effect on the growth of the swell and the neck of the jet. For small evaporation rates the swell grows in the same manner as that for the nonevaporation case. As the evaporation rate is increased, the evaporation mechanism begins to dominate, and the jet breakup time and the satellite/main drop sizes both decrease. Eventually, when an evaporation rate of 0.05 is reached no satellites are produced for the range of Reynolds and wave numbers considered. The growth rate also increases as the evaporation rate is increased. For very large evaporation rate the growth of the disturbance becomes very nonlinear and can no longer be predicted by the logarithmic amplitude of the swell-neck curve.

\section{References}

${ }^{1}$ Bidone, G., "Experiences sur la Forme et sur la Direction des Veines et des Courants d'eau Lances par Diverses Ouvertures," Imprimerie Royale, Turin, 1829, pp. 1-136.

${ }^{2}$ Magnus, G., "On the Swellings Formed in Jets Issuing from Circular Orifices," Philosophical Magazine, Vol. 18, 1859, pp. 161-183.

${ }^{3}$ Rayleigh, L., "On the Instability of Jets," Proceedings of the London Mathematical Society, Vol. 10, 1879, pp. 4-13.

${ }^{4}$ Rayleigh, L., "Further Observations upon Liquid Jets," Proceedings of the London Mathematical Society, Vol. 34, 1882, pp. 130-145.

${ }^{5}$ Chandrasekhar, S., Hydrodynamic and Hydromagnetic Stability, Clarendon, Oxford, 1961, pp. 537-540.

${ }^{6}$ Caulk, D. A., "Some Two and One Dimensional Problems in Fluid Mechanics by a Direct Approach," Ph.D. Dissertation, Engineering Science, Univ. of California, Berkeley, CA, June 1976.

${ }^{7}$ Caulk, D. A., and Naghdi, P. M., "The Influence of Twist on the Motion of a Straight Elliptical Jet," Univ. of California, Dept. of Mechnical Engineering, Rept. UCB/AM-77-5, Berkeley, CA, June 1977.

${ }^{8}$ Caulk, D. A., and Naghdi, P. M., "The Onset of Breakup in Inviscid and Viscous Jets," Univ. of California, Dept. of Mechnical Engineering, Rept. UCB/AM-78-3, Berkeley, CA, July 1978.

${ }^{9}$ Yuen, M. C., "Non-Linear Capillary Instability of a Liquid Jet," Journal of Fluid Mechanics, Vol. 33, Pt. 1, 1968, pp. 151-163.

${ }^{10}$ Nayfeh, A. H., and Hassan, S. D., "The Method of Multiple Scales and Nonlinear Dispersive Waves," Journal of Fluid Mechanics, Vol. 48, Pt. 3, 1971, pp. 463-475.

${ }^{11}$ Lafrance, P., "Nonlinear Break up of a Laminar Liquid Jet," Physics of Fluids, Vol. 18, No. 4, 1975, pp. 428-432.

${ }^{12}$ Pimbley, W. T., "Drop Formation from a Liquid Jet: A Linear OneDimensional Analysis Consdered as a Boundary Value Problem," IBM Journal of Research and Development, Vol. 20, No. 2, 1976, pp. 148-156.

${ }^{13}$ Chaudhary, K. C., and Redekopp, L. G., "The Nonlinear Capillary Instability of a Liquid Jet. Part 1. Theory," Journal of Fluid Mechanics, Vol.96, Pt. 2, 1980, pp. 257-274.

${ }^{14}$ Torpey, P. A., "A Nonlinear Theory for Describing the Propagation of Disturbances on a Capillary Jet," Physics of Fluids A, Vol. 1, No. 4, 1989, pp. 661-671.

${ }^{15}$ Green, A. E., "On the Non-Linear Behavior of Fluid Jets," International Journal of Engineering Science, Vol. 14, No. 1, 1976, pp. 49-63.

${ }^{16}$ Mansour, N. N., and Lundgren, T. S., "Satellite Formation in Capillary Jet Breakup,” Physics of Fluids A, Vol. 2, No. 7, 1990, pp. 1141-1144.

${ }^{17}$ Fromm, J. E., "Numerical Calculation of the Fluid Dynamics of Dropon-Demand," IBM Journal of Research and Development, Vol. 28, No. 3, 1984, pp. 322-333.

${ }^{18}$ Shokoohi, F., and Elrod, H. G., "Numerical Investigation of the Disintegration of Liquid Jets," Journal of Computational Physics, Vol. 71, No. 2, 1987, pp. 324-342.

${ }^{19}$ Ashgriz, N., and Mashayek, F., "Temporal Analysis Capillary Jet Breakup," Journal of Fluid Mechanics, Vol. 291, 1995, pp. 163-190.

${ }^{20}$ Lian, Z. W., and Reitz, R. D., "The Effect of Vaporization and Gas Compressibility on Liquid Jet Atomization," Atomization and Sprays, Vol. 3 , 1993, pp. 249-264.

${ }^{21}$ Prosperetti, A., and Plesset, M. S., "The Stability of an Evaporating Liquid Surface," Physics of Fluids, Vol. 27, No. 7, 1984, pp. 1590-1602.

${ }^{22}$ Mashayek, F., and Ashgriz, N., "A Height Flux Method for Simulating Free Surface Flows and Interfaces," International Journal for Numerical Methods in Fluids, Vol. 17, No. 12, 1993, pp. 1035-1054.

S. K. Aggarwal Associate Editor 